\title{
Manage comfort preferences conflicts using a multi-agent system in an Adaptive Environment System
}

\author{
Pedro Filipe Oliveira ${ }^{12}$, Paulo Novais ${ }^{1}$, and Paulo Matos ${ }^{2}$ \\ 1 Algoritmi Centre/University of Minho, Department of Informatics, Braga, Portugal \\ 2 Instituto Politécnico de Bragança, Campus de Santa Apolónia, 5300-253 Bragança, \\ Portugal
}

\begin{abstract}
Managing comfort preferences conflicts of the different users and locals on an IoT adaptive system is a actual problem, this paper proposes a protocol and hierarchical rules to develop a multi-agent system to achieve a Adaptive Environment System that solves the management of conflicts in an autonomous way for the users and interdependent of the user schedules and routines.
\end{abstract}

Keywords: adaptive-system, AmI, multi-agent, IoT, conflicts

\section{Introduction}

As in any environment where different users are present, there will be a native conflict of interest regarding the most diverse issues. Also in this case of developing an intelligent environment adaptable to the comfort preferences of each user, this problem will be present. It is well known that in everyday life, we deal with people with different comfort preferences, both in terms of temperature or humidity values, as well as in terms of musical or video tastes, etc. This is more critical, as these values interfere with people's well-being, or even their health, namely in terms of allergies, diseases, etc.

In this project, a protocol was created, which allows to resolve as much as possible this type of conflicts, thus trying to reach a optimum preference value, which satisfies as much as possible the majority of users present in the environment. This work resulted in the complete specification of an architecture that supports the solution found, to solve the presented problem. It will now be implemented, tested and validated using real case studies, so as to gather statistical information to assess its effectiveness and performance in the context of application. This work aims to give continuity and finalize the doctoral work presented in previous editions [6][7][9][8].

\section{Materials and Methods}

Figure 1, shows the scenario where we develop this work. It can be seen the user who through its different devices (smartphone, wearable, and other compatible) 
communicates with the system, and for that can be used different technologies, like Near Field Communication (NFC) [10], Bluetooth Low Energy (BLE) [1] or Wi-Fi Direct [2]. After, the system communicates with the Cloud, to validate the information, and the system will perform the management of different components and actuators in the environment (climatization systems, security systems, other smart systems).

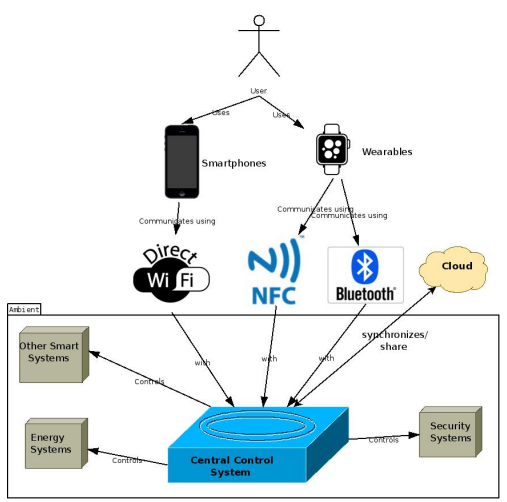

Fig. 1: Problem Statement [6]

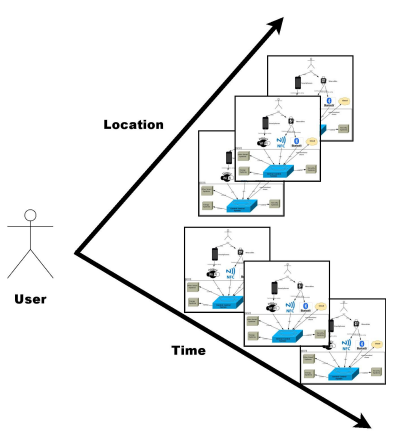

Fig. 2: Contextualization of Time/Environment Dimensions [6]

\section{Results}

This section presents the technologies used in this project for the development of the entire multi-agent system applied to AmI.

At figure 3 is represented the different architecture layers, the agent that represents the local system receives its information, namely the security information (maximum values of temperature, gases, and others). Also for each user present at the local, there will be an agent who represents him, he will receive information about the user preferences from the central system, that will be used for the negotiation process.

The negotiation process will then be made up of the local system agent and each of the users agents present at the local. The negotiation result of will then be passed on to the different actuators present in the local.

In the course of this project, the environments the focuses will be mainly on domestic/family, professional environments (workplaces) and public spaces, where a large number of people are usually present.

One of the rules used for conflict resolution was the hierarchy of preferences. Starting with family contexts, it was taken into account to maximize the preference value of adult elements (parents) over the children, in a ratio of 1 to 0.75. Another hierarchy is the preference value of the space if it exists, in this case a 


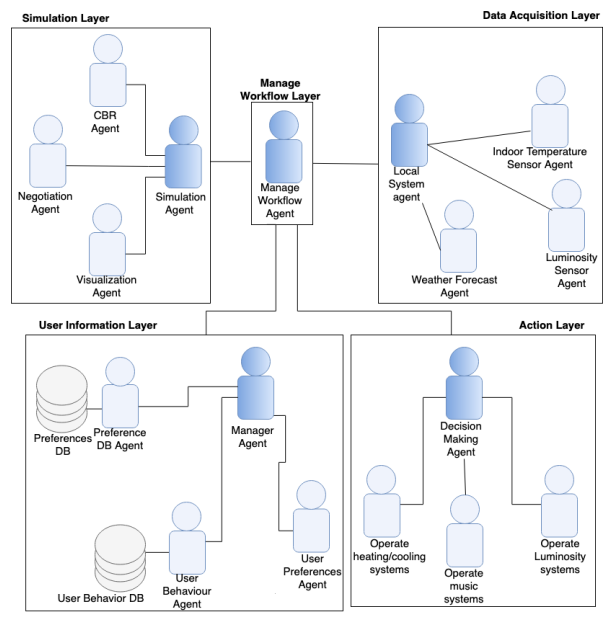

Fig. 3: Architecture of the multi-agent system [9][4][3][5]

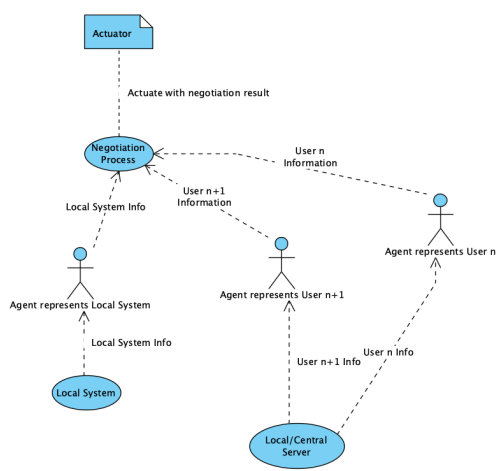

Fig. 4: AmI System - Use Case diagram

proportion of 1.5 will be used. These cases may exist in spaces where there is some conditioning, such as kitchens/wc, or other spaces that have some type of conditioning.

The proportions described and used for the rules are detailed in Table 1.

In the professional context, the proportion values are also defined in a hierarchical way, and in this context the professional hierarchy of space will be used, as well as the space preference value if it exists. The proportions described are detailed in Table 2.

Regarding public/social spaces, the predominant value will obviously be the space value with a proportion of 2 , and each user will have a proportion of 0.15 , as in these spaces it is natural that there is little variation in the values, derived by the high movement of people. The proportions described are detailed in Table 3 . The formula used to achieve the optimum preference value to the different spaces is the following:

$$
\text { prefValue }=\frac{\sum_{u s e r=1}^{n} \text { userPref } \times \text { user HierProportion }+(\text { spacePref } \times \text { spaceProportion })}{\sum_{u s e r=1}^{n} \text { userHierProportion }+ \text { spaceProportion }}
$$

\begin{tabular}{|c|c|}
\hline Type & Proportion \\
\hline Adult & 1 \\
\hline Child & 0,75 \\
\hline Visitor & 1 \\
\hline Space & 1,5 \\
\hline
\end{tabular}

Table 1: Type of users and proportions - Home space

\begin{tabular}{|c|c|}
\hline Type & Proportion \\
\hline Hierarchy_1 & $(100-1)$ \\
\hline Hierarchy_2 & $(100-2)$ \\
\hline Hierarchy_n & $(100-\mathrm{n})$ \\
\hline Space & 150 \\
\hline
\end{tabular}

Table 2: Type of users and proportions - Work space

\begin{tabular}{|c|c|}
\hline Type & Proportion \\
\hline User_1 & 0,15 \\
\hline User_2 & 0,15 \\
\hline User_n & 0,15 \\
\hline Space & 2 \\
\hline
\end{tabular}

Table 3: Type of users and proportions - Public/Social space 


\section{Discussion and Conclusions}

This work resulted in the complete specification of an architecture that supports the solution found, to solve the presented problem. The agent system model is fully developed. At this stage the agent layer was developed, implemented, and is now in a testing phase at the testing environment developed for this project. Now It will be tested and validated using real case studies, so as to gather statistical information to assess its effectiveness and performance in the context of application.

For future work, the result tests will be evaluated, in order to verify the model optimization possibilities and implementation.

\section{Acknowledgments}

This work has been supported by FCT - Fundação para a Ciência e Tecnologia within the Project Scope: UID/CEC/00319/2019.

\section{References}

1. Bluetooth, S.: Bluetooth core specification version 4.0. Specification of the Bluetooth System (2010)

2. Camps-Mur, D., Garcia-Saavedra, A., Serrano, P.: Device-to-device communications with wi-fi direct: overview and experimentation. Wireless Communications, IEEE 20(3), 96-104 (2013)

3. González-Briones, A., Chamoso, P., De La Prieta, F., Demazeau, Y., Corchado, J.M.: Agreement technologies for energy optimization at home. Sensors 18(5), 1633 (2018)

4. González-Briones, A., De La Prieta, F., Mohamad, M.S., Omatu, S., Corchado, J.M.: Multi-agent systems applications in energy optimization problems: A stateof-the-art review. Energies 11(8), 1928 (2018)

5. González-Briones, A., Prieto, J., De La Prieta, F., Herrera-Viedma, E., Corchado, J.M.: Energy optimization using a case-based reasoning strategy. Sensors 18(3), $865(2018)$

6. Oliveira, P., Matos, P., Novais, P.: Behaviour analysis in smart spaces. In: 2016 Intl IEEE Conferences on Ubiquitous Intelligence \& Computing, Advanced and Trusted Computing, Scalable Computing and Communications, Cloud and Big Data Computing, Internet of People, and Smart World Congress (UIC/ATC/ScalCom/CBDCom/IoP/SmartWorld). pp. 880-887. IEEE (2016)

7. Oliveira, P., Novais, P., Matos, P.: Challenges in smart spaces: Aware of users, preferences, behaviours and habits. In: International Conference on Practical Applications of Agents and Multi-Agent Systems. pp. 268-271. Springer (2017)

8. Oliveira, P., Novais, P., Matos, P.: Generating real context data to test user dependent systems-application to multi-agent systems. In: International Conference on Practical Applications of Agents and Multi-Agent Systems. pp. 180-187. Springer (2019)

9. Oliveira, P.F., Novais, P., Matos, P.: A multi-agent system to manage users and spaces in a adaptive environment system. In: International Conference on Practical Applications of Agents and Multi-Agent Systems. pp. 330-333. Springer (2019)

10. Want, R.: Near field communication. IEEE Pervasive Computing 0(3), 4-7 (2011) 